\title{
Effects of resistance training on classic and specific bioelectrical impedance vector analysis in elderly women
}

\author{
David H. Fukuda a,*, Jeffrey R. Stout ${ }^{\text {a }}$, Jordan R. Moon ${ }^{\text {b }}$, Abbie E. Smith-Ryan ${ }^{\text {c }}$, \\ Kristina L. Kendall ${ }^{\text {, }}$, Jay R. Hoffman ${ }^{\text {a }}$ \\ ${ }^{a}$ Institute of Exercise Physiology \& Wellness, University of Central Florida, Orlando, FL, United States \\ b United States Sports Academy, Daphne, AL, United States \\ ' Exercise and Sport Science, University of North Carolina, Chapel Hill, Chapel Hill, NC, United States \\ ${ }^{\mathrm{d}}$ Bodybuilding.com, Boise, ID, United States
}

\section{A R T I C L E I N F O}

\section{Article history:}

Received 19 September 2015

Received in revised form 6 November 2015

Accepted 1 December 2015

Available online 2 December 2015

\section{Keywords:}

Aging

Body composition

Bioelectrical impedance

Resistance training

BIVA

Phase angle

\begin{abstract}
A B S T R A C T
Raw bioelectrical impedance analysis (BIA) data [resistance $(\mathrm{R})$; reactance $(\mathrm{Xc})$ ] through bioelectrical impedance vector analysis (BIVA) and phase angle ( $\mathrm{PhA}$ ) have been used to evaluate cellular function and hydration status. The purpose of this investigation was to examine the effects of resistance training (RT) on classic and specific BIVA in elderly women. Twenty women (mean \pm SD; age: $71.9 \pm 6.9$ years; BMI: $24.5 \pm 3.0 \mathrm{~kg} \mathrm{~m}^{-2}$ ) completed a 6-month RT program. Whole-body, single-frequency BIA, body geometry, and leg strength (5RM) measures were completed at baseline (t0), 3 months ( $\mathrm{t} 3$ ), and 6 months ( $\mathrm{t} 6$ ). The mean impedance vector displacements were compared using Hotelling's $\mathrm{T}^{2}$ test to evaluate changes in $\mathrm{R}$ and Xc relative to height (R/ht; Xc/ht) or body volume $\left(\mathrm{R}_{\mathrm{sp}} ; \mathrm{Xc}_{\mathrm{sp}}\right)$ estimated from the arms, legs, and trunk. 5RM, PhA, and BIVA variables were compared using ANOVA. PhA improved at $\mathrm{t} 6(\mathrm{p}<0.01)$, while 5RM improved at $\mathrm{t} 3$ and $\mathrm{t} 6(\mathrm{p}<0.01)$. Using classic BIVA, 6 months $\left(\mathrm{T}^{2}=31.6 ; \mathrm{p}<0.01\right)$, but not 3 months of $\mathrm{RT}\left(\mathrm{T}^{2}=4.5 ; \mathrm{p}=0.20\right)$, resulted in significant vector migration. Using specific BIVA, 6 months ( $\mathrm{T}^{2}=24.4 ; \mathrm{p}<0.01$ ), but not 3 months of $\mathrm{RT}\left(\mathrm{T}^{2}=5.5 ; \mathrm{p}=0.10\right.$ ), also resulted in significant vector migration. 5RM was correlated to both $\mathrm{PhA}(\mathrm{r}=0.48-56)$ and $\mathrm{Xc}_{\mathrm{sp}}(\mathrm{r}=0.45-53)$ at all time points. Vector displacements were likely the result of improved cellular integrity $\left(\mathrm{Xc}_{\mathrm{sp}}\right)$ and cellular health $(\mathrm{PhA})$.

(C) 2015 Elsevier Inc. All rights reserved.
\end{abstract}

\section{Introduction}

The raw values from bioelectrical impedance analysis (BIA), resistance $(\mathrm{R})$ and reactance $(\mathrm{Xc})$, are used to determine phase angle, an indicator of cellular health/function, which is related to functional status and impaired prognosis with disease (Norman et al., 2012). Graphical interpretation of $\mathrm{R}$, a measure of cellular hydration status, and Xc, a measure of cell membrane integrity, termed bioelectrical impedance vector analysis (BIVA), has been proposed as a method of body composition evaluation in older adults (Lukaski, 2013). Traditionally, vectors from raw BIA data have been determined relative to an individual's height in order to account for differences in conductor length (Piccoli et al., 1994). Specific BIVA standardizes the vector values with consideration for the conductor volume by utilizing a variation of Ohm's law while accounting for the cross-sectional areas of the upper arm, calf,

Abbreviations: BIVA, bioelectrical impedance vector analysis; $\mathrm{PhA}$, phase angle; 5RM five-repetition maximum leg extension strength; $t 0$, baseline; $t 3,3$ months of resistance training; $t 6,6$ months of resistance training; $\mathrm{R}_{\mathrm{sp}}$, specific resistance; $\mathrm{Xc}_{\mathrm{sp}}$, specific reactance.

* Corresponding author at: University of Central Florida, 12494 University Blvd, ED 320R, Orlando, FL 32816, United States.

E-mail address: david.fukuda@ucf.edu (D.H. Fukuda). and waist (Marini et al., 2013). In particular, the estimation of conductor cross-sectional area through the measurement of circumferences with specific BIVA was proposed to overcome the potential limitation of accounting for solely conductor length with classic BIVA (Buffa et al., 2013).

The clinical application of BIVA methodologies has been highlighted due to the direct analysis of the bioelectrical properties of human tissue, which limits the potential error introduced by prediction equations used to estimate body composition (Lukaski, 2013). Classic BIVA has shown to have utility with regard to the evaluation of nutritional and/ or hydration status (Piccoli et al., 2014), while specific BIVA may be a better indicator of two-component body composition (Buffa et al., 2014) with the potential ability to distinguish between older adults with sarcopenia and sarcopenic-obesity (Marini et al., 2012).

Further, cross-sectional data has shown age-related changes in whole-body BIVA variables, with increases in phase angle and decreases in Xc throughout the progression of adolescence (Koury et al., 2014), but decreases in both with advanced age (Saragat et al., 2014), and differences between individuals of varying training status/performance levels (Micheli et al., 2014) and sporting activities (Koury et al., 2014). However, limited longitudinal data concerning changes in BIVA variables exist. These investigations have focused on whole-body BIA 
during pregnancy (Lukaski et al., 2007) and segmental BIA during recovery from injury (Nescolarde et al., 2011).

Resistance training in older adults has shown to be an efficacious intervention which results in improvements in both muscle mass and function (Peterson et al., 2011). The measurement of BIA in older adults has been proposed to evaluate the presence of sarcopenia (Marini et al., 2012) and improvements in BIA-derived fat-free mass have been reported following resistance training in elderly men and women (Moon et al., 2013). However, the application of BIVA to resistance training in older adults has not been examined. Therefore, the purpose of this investigation was to examine the effects of resistance training on phase angle and to compare classic and specific BIVA in elderly women.

\section{Materials and methods}

Unpublished strength, anthropometric, and bioelectrical data from 20 healthy, ambulatory women (mean \pm SD; age: $71.9 \pm 6.9$ years; body mass index: $24.5 \pm 3.0 \mathrm{~kg} \mathrm{~m}^{2}$ ) that were involved in an institutional review board approved longitudinal study (Stout et al., 2013) and completed an informed consent as well as all of the requisite testing measures were used in this investigation. All participants completed a three times weekly, 6-month full-body progressive resistance training program. Testing procedures were completed at baseline (t 0 ), 3 months (t3), and 6 months (t6) (Table 1). Leg extension strength was assessed using a five-repetition (5RM) test. The circumferences of the upper arm (at the midpoint between the acromion and olecranon processes), thigh (at the midpoint between the inguinal crease and proximal border of the patella), calf (at the point of maximal girth), and waist (at the level of the navel) were measured in triplicate with an anthropometric measuring tape. The upper arm, thigh, and calf values were used to estimate the cross-sectional area of the arms, legs, and trunk, respectively, using the formula: area $=$ circumference $^{2} / 4 \pi$, while waist circumferences were used as a structural outcome to evaluate the potential changes in the functional outcome of leg strength. Hydration status with urine specific gravity values less than 1.030 was verified using handheld refractometry (Model CLX-1; VEE GEE Scientific, Inc. Kirkland, WA, USA).

Whole-body, tetrapolar, single-frequency $(50 \mathrm{~Hz})$ BIA (Quantum II, RJL Systems, Clinton Township, MI), with electrodes (Dual Tab, ImpediMed Limited, Queensland, Australia) placed at the hand and foot, was used to determine R $(\Omega)$, Xc $(\Omega)$, impedance $(Z ; \Omega)$, and phase angle $\left({ }^{\circ}\right)$. The BIA device was calibrated with a $500 \Omega$ test resistor (to verify $\mathrm{R}$ of $500 \pm 5 \Omega$ and Xc of $0 \pm 5 \Omega$ ) per manufacturer

Table 1

Comparison of anthropometric, strength, bioelectrical values at baseline (t0), after 3 months of resistance training ( $\mathrm{t} 3$ ), and after 6 months of resistance training ( $\mathrm{t} 6$ ).

\begin{tabular}{llll}
\hline & t0 & t3 & t6 \\
\hline Weight $(\mathrm{kg})$ & $63.6 \pm 9.8$ & $63.6 \pm 10.0$ & $64.1 \pm 10.1$ \\
Calf circumference $(\mathrm{cm})$ & $34.7 \pm 3.2$ & $34.5 \pm 3.2$ & $34.5 \pm 3.3$ \\
Arm circumference $(\mathrm{cm})$ & $27.0 \pm 2.7$ & $27.1 \pm 2.7$ & $27.5 \pm 2.7$ \\
Waist circumference $(\mathrm{cm})$ & $76.4 \pm 7.9$ & $75.5 \pm 8.5$ & $76.7 \pm 8.2^{\dagger}$ \\
Thigh circumference $(\mathrm{cm})$ & $47.6 \pm 4.4$ & $47.7 \pm 4.5$ & $50.1 \pm 4.4^{*}$, \\
Leg extension 5-RM $(\mathrm{kg})$ & $30.3 \pm 8.0$ & $42.6 \pm 10.6$ & $59.3 \pm 12.8^{*, \dagger}$ \\
R; resistance $(\Omega)$ & $603.7 \pm 53.4$ & $604.0 \pm 62.5$ & $599.3 \pm 69.2$ \\
Xc; reactance $(\Omega)$ & $50.6 \pm 7.6$ & $51.6 \pm 7.0$ & $52.9 \pm 9.2$ \\
Z; impedance $(\Omega)$ & $376.9 \pm 45.4$ & $377.1 \pm 49.6$ & $374.3 \pm 55.2$ \\
Phase angle $\left(^{\circ}\right)$ & $4.80 \pm 0.61$ & $4.89 \pm 0.53$ & $5.04 \pm 0.67^{*, \dagger}$ \\
R/ht $(\Omega / \mathrm{m})$ & $376.9 \pm 45.4$ & $377.1 \pm 49.6$ & $374.3 \pm 55.2$ \\
Xc/ht $(\Omega / \mathrm{m})$ & $31.6 \pm 5.5$ & $32.2 \pm 5.1$ & $33.0 \pm 6.6$ \\
r R/ht-Xc/ht & $0.650 \mathrm{p}<0.01$ & $0.715 \mathrm{p}<0.01$ & $0.723 \mathrm{p}<0.01$ \\
Rsp $(\Omega \cdot \mathrm{cm})$ & $395.5 \pm 48.6$ & $391.3 \pm 49.3$ & $397.2 \pm 59.3$ \\
Xcsp $(\Omega \cdot \mathrm{cm})$ & $33.2 \pm 5.8$ & $33.4 \pm 5.3$ & $35.1 \pm 7.3^{*, \dagger}$ \\
r Rsp-Xcsp & $0.667 \mathrm{p}<0.01$ & $0.717 \mathrm{p}<0.01$ & $0.756 \mathrm{p}<0.01$ \\
\hline
\end{tabular}

$\mathrm{r}=$ Pearson product moment correlation.

* Denotes a significant difference from baseline $(\mathrm{p} \leq 0.05)$.

$\dagger$ Denotes a significant difference from 3 months $(\mathrm{t} 3)(\mathrm{p} \leq 0.05)$. guidelines. Specific resistance $\left(R_{s p}\right)$ and reactance $\left(X_{s p}\right)$ values were calculated by multiplying $\mathrm{R}$ and $\mathrm{Xc}$ by correction factors [quantified as the estimated cross-sectional area (or $0.45 \times$ arm area $+0.10 \times$ waist area $+0.45 \times$ calf area) divided the length of the body modeled as a conductor (or $1.1 \times$ height)] as outlined by Marini et al. (2013), while classic BIVA values were calculated relative to standing height $(\mathrm{R} / \mathrm{ht}$ and $\mathrm{Xc} / \mathrm{ht}$ ).

The resistance training program has been previously reported (Stout et al., 2013). Exercises included hack squat, bilateral leg press, leg extension, bench press and lat pull down. Participants were asked to complete 8-12 repetitions of each exercise, in a laboratory, supervised by trained research personnel. Each exercise was separated by a 2-5 min recovery period. If subjects completed 12 repetitions for the last set of an exercise for two consecutive lifting sessions, weight was increased by at least $2.5-10 \%$ depending on the exercise.

\subsection{Statistical analysis}

Strength and BIVA values were compared using repeated measures analysis of variance with Bonferroni post-hoc tests. The mean impedance vector displacements from classic and specific BIVA were compared with BIVA software (Piccoli and Pastori, 2002) using Hotelling's $\mathrm{T}^{2}$ test to compare the changes between $\mathrm{R}_{\mathrm{sp}}$ and $\mathrm{Xc}_{\mathrm{sp}}$. The relationship between BIVA values and strength (5RM) as well as the relationship between changes $(\Delta \mathrm{t} 6-\mathrm{t} 0)$ in these variables were analyzed using Pearson correlations.

\section{Results}

\subsection{Anthropometrics and strength}

The anthropometric and strength values are listed in Table 1. No significant changes in body mass $(\mathrm{p}=0.246)$, upper arm circumference ( $p=0.06)$ or calf circumference $(p=0.471)$ were reported. Significant training effects were shown for waist circumference $(p=0.049)$, thigh circumference ( $p \leq 0.01)$, and leg extension 5RM $(p<0.01)$. Waist circumference increased from t3 to t6 $(\Delta: 1.3 \mathrm{~cm} ; \mathrm{p}<0.01)$, thigh circumference increased from to to t6 $(\Delta: 2.5 \mathrm{~kg} ; \mathrm{p} \leq 0.01)$ and from t3 to t6 ( $\Delta$ : $2.4 \mathrm{~kg} ; \mathrm{p} \leq 0.01$ ), while leg extension $5 \mathrm{RM}$ increased at both $\mathrm{t} 3(\Delta$ : $12.4 \mathrm{~kg} ; \mathrm{p}<0.01)$ and t6 $(\Delta: 29 \mathrm{~kg} ; \mathrm{p}<0.01)$ and from t3 to t6 $(\Delta$ : $16.7 \mathrm{~kg} ; \mathrm{p}<0.01)$.

\subsection{Bioelectrical data}

The bioelectrical data are listed in Table 1. Raw Z $(p=0.806)$ and $R$ $(\mathrm{p}=0.770)$ values showed nonsignificant decreases, while raw Xc values showed a nonsignificant increase $(p=0.057)$, throughout the training program. Significant training effects were shown for phase angle $(\mathrm{p}<0.01)$ with improvements from to to t6 $\left(\Delta: 0.25^{\circ} ; \mathrm{p}<0.01\right)$ and $\mathrm{t} 3$ to $\mathrm{t} 6\left(\Delta: 0.16^{\circ} ; \mathrm{p}=0.012\right)$, but not from to to $\mathrm{t} 3\left(\Delta: 0.09^{\circ} ; \mathrm{p}=\right.$ $0.230)$. Nonsignificant changes in $\mathrm{R} / \mathrm{ht}(\mathrm{p}=0.807), \mathrm{Xc} / \mathrm{ht}(\mathrm{p}=0.056)$, and $R_{s p}(p=0.309)$ were reported. Significant training effects were found for $\mathrm{Xc}_{\mathrm{sp}}(\mathrm{p}=0.001)$, with in an increase from t0 to t6 $(\Delta$ : $1.9 \Omega * \mathrm{~cm} ; \mathrm{p}=0.011)$ and t3 to t6 $(\Delta: 1.7 \Omega * \mathrm{~cm} ; \mathrm{p}=0.032)$, but not from to to $\mathrm{t} 3(\Delta: 0.2 \Omega * \mathrm{~cm} ; \mathrm{p}>0.05)$.

\subsection{Impedance vector displacement}

With classic BIVA, the completion of 6 months $\left(\mathrm{T}^{2}=31.6 ; \mathrm{p}<0.01\right)$, but not 3 months of resistance training $\left(\mathrm{T}^{2}=4.5 ; \mathrm{p}=0.20\right)$, resulted in significant vector migration where $95 \%$ confidence ellipses for the mean differences in $\mathrm{R} / \mathrm{ht}$ and $\mathrm{Xc} / \mathrm{ht}$ did not cross the origin (Fig. 1A). When using specific BIVA, 6 months $\left(\mathrm{T}^{2}=24.4 ; \mathrm{p}<0.01\right)$, but not 3 months of resistance training $\left(\mathrm{T}^{2}=5.5 ; \mathrm{p}=0.10\right)$, also resulted in significant vector migration (Fig. 1B). In addition, the impedance ellipses for classic and specific BIVA at $\mathrm{t} 0, \mathrm{t} 3$, and $\mathrm{t} 6$ are shown in Fig. 2. 

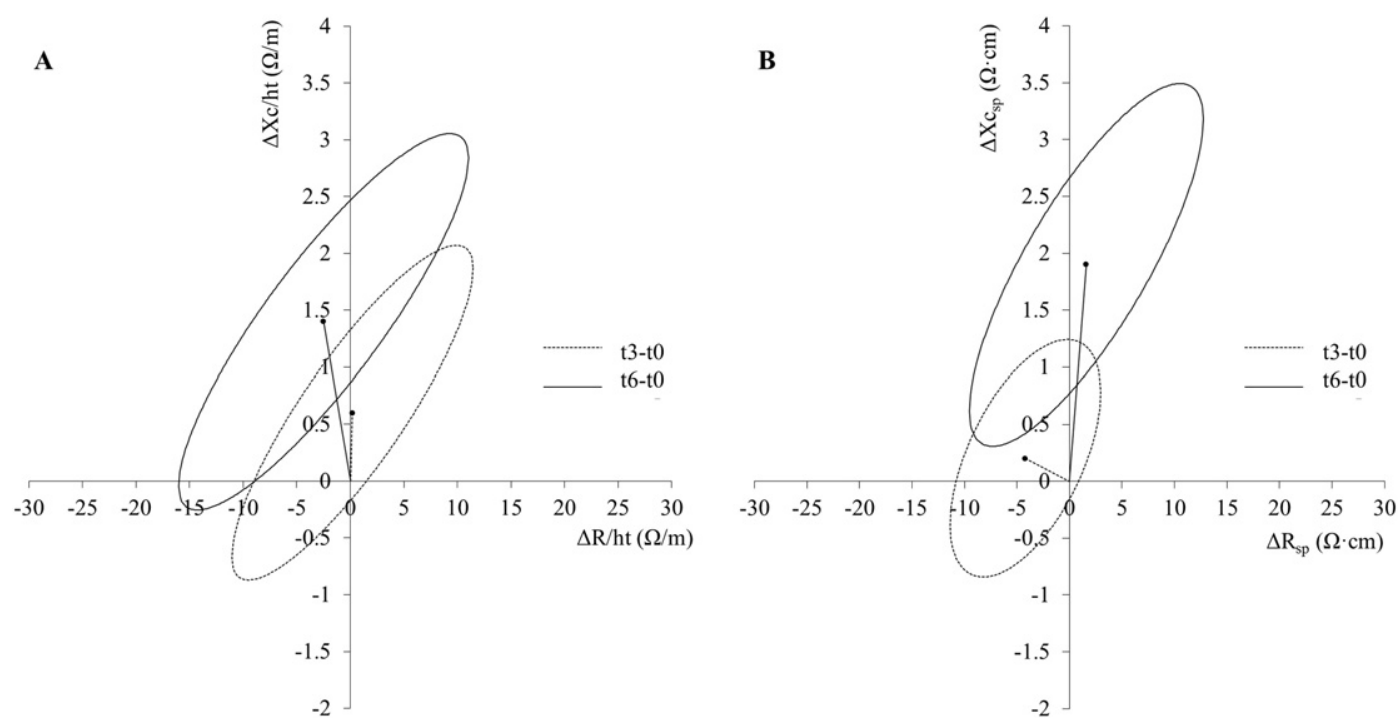

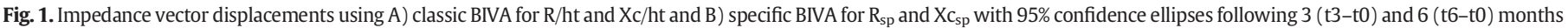
of resistance training.

\subsection{Correlations}

Phase angle and 5RM were significantly associated at t0 $(r=0.478$; $\mathrm{p}=0.033)$, t3 $(\mathrm{r}=0.555 ; \mathrm{p}=0.011)$, and $\mathrm{t} 6(\mathrm{r}=0.533 ; \mathrm{p}=0.015)$, while $\mathrm{Xc}_{\mathrm{sp}}$ and $5 \mathrm{RM}$ also showed significant positive correlation at all time points ( $\mathrm{t} 0: \mathrm{r}=0.453 ; \mathrm{p}=0.045 ; \mathrm{t} 3: \mathrm{r}=0.531 ; \mathrm{p}=0.016$; $\mathrm{t}$ : $r=0.528 ; p=0.017$ ). No relationships between the changes ( $t 6-t 0)$ in strength and bioelectrical data were found.

\section{Discussion}

The primary finding of this investigation demonstrated differential patterns of adaptation reflected by classic and specific BIVA in elderly women following prolonged resistance training. Qualitative interpretation of the impedance vector displacement graph using classic BIVA revealed similar contributions of $\mathrm{R} / \mathrm{ht}$ and $\mathrm{Xc} / \mathrm{ht}$ to the changes at 6 months, while specific BIVA demonstrated that changes at 3 months were largely due to decreased $R_{s p}$ while changes at 6 months were due to an increase in $\mathrm{Xc}_{\mathrm{sp}}$. This observation supports the potential need to account for conductor volume (as a function of segmental cross-sectional area), as opposed to conductor length (as a function of stature), when evaluating bioelectrical data. Therefore, specific BIVA may provide conflicting values as compared to classic BIVA when evaluating changes following resistance training.

Nonsignificant decreases in $\mathrm{R}_{\mathrm{sp}}$ at 3 months and $\mathrm{R} / \mathrm{ht}$ over the course of the resistance training program may reflect subtle improvements in body composition (Buffa et al., 2014), as a result of decreased fat mass and/or increased glycogen stores, and cellular hydration. Reactance (Xc) measured from BIA has been shown to be related to body cell mass in older adults (Dittmar and Reber, 2001). The increase in $\mathrm{Xc}_{\mathrm{sp}}$ at 6 months in the current investigation and its relationship with strength suggest that this measure may be related to cellular adaptation. Furthermore, the enhanced training status of the current study participants at the end of the exercise intervention can be compared to crosssectional differences in Xc between football (Xc/ht: $37.39 \pm 3.82 \Omega$ ) and basketball athletes (Xc/ht: $28.75 \pm 4.87 \Omega$ ) (Nescolarde et al., 2011). Phase angle has previously shown to be significantly related to skeletal muscle mass (Buffa et al., 2013) and a predictor of both general health status and muscle strength (Norman et al., 2012, 2015). The
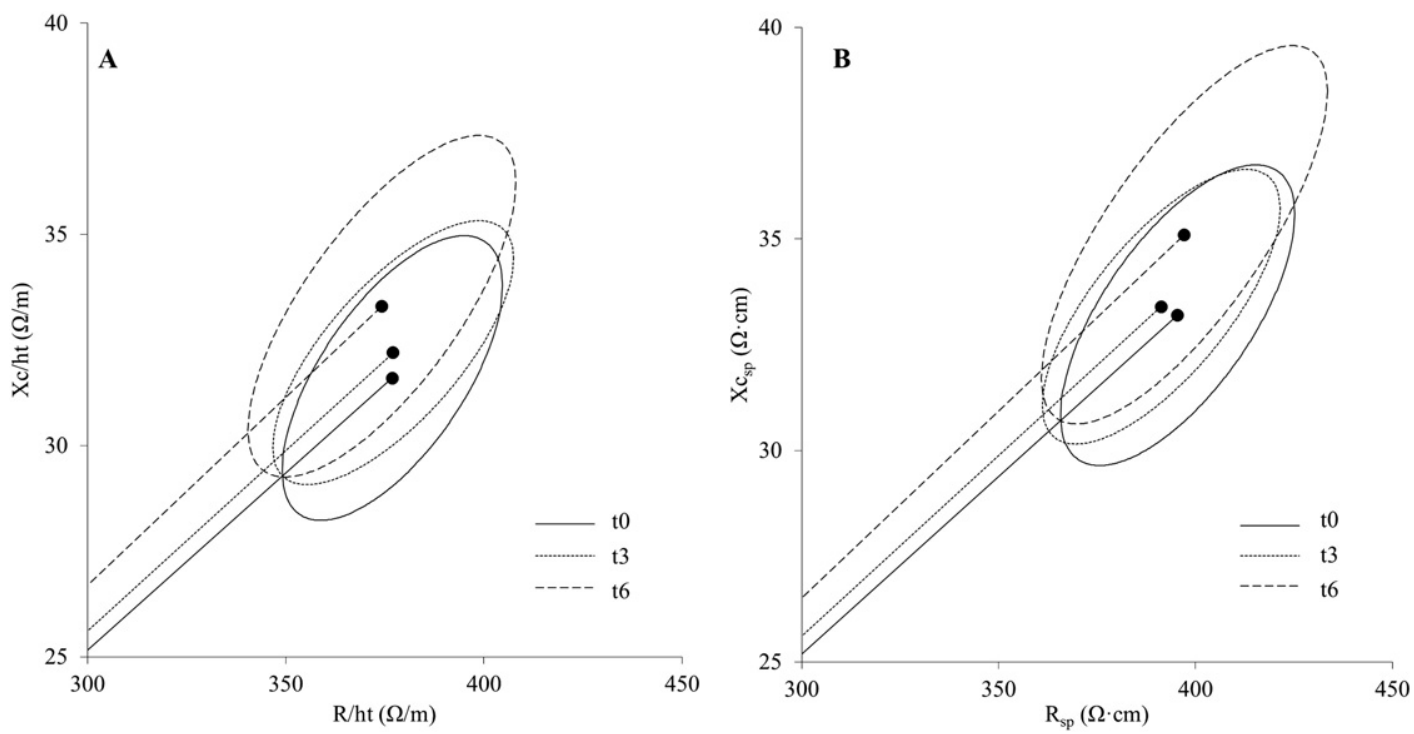

Fig. 2. 95\% confidence impedance ellipses for A) classic BIVA and B) specific BIVA at baseline (t0) and following 3 (t3) and 6 (t6) months of resistance training. 
currently reported improvements appear to be in opposition to the agerelated decreases in phase angle and Xc (Saragat et al., 2014). The results of the current study showed that phase angle and $\mathrm{Xc}_{\mathrm{sp}}$ were significantly associated with leg extension strength at all time points. The functional improvements in leg strength were supported by increased thigh circumference following 6 months of resistance training.

The qualitative distinction between contributions of $\mathrm{R}_{\mathrm{sp}}$ and $\mathrm{X} \mathrm{c}_{\mathrm{sp}}$ by specific BIVA at 3 and 6 months appears to be consistent with the existing literature in regards to strength training adaptations. The satellite cell pool has shown to be increased after 3 months of resistance training in older men and women (70-82 years) (Mackey et al., 2007). Furthermore, a meta-analysis by Peterson et al. (2011) reported a significant effect for resistance training on lean body mass in elderly men and women from interventions lasting on average 20.5 weeks.

\section{Conclusions}

The bioelectric properties determined from BIA and evaluated with both classic and specific BIVA changed as a result of 6 months of resistance training in elderly women. Nonetheless, the findings after a 6month training program highlight the maintenance of baseline cellular hydration with increasing cellular membrane integrity. Altered vector displacements were likely the result of improved cellular integrity $\left(\mathrm{Xc}_{\mathrm{sp}}\right)$ and cellular health (phase angle). While the current results cannot be used to identify a preferred method, classic and specific BIVA provide diverging information about the initial process of adaptation with regard to cellular hydration. Additional research utilizing larger sample sizes comparing classic and specific BIVA methodologies to "gold standard" body composition and cellular function measures to evaluate concurrent validity is needed. Furthermore, exploration of segmental BIA (arms, legs, trunk) with vector analysis and site-specific changes in strength or performance following resistance training may be of particular interest. Finally, researchers should consider reporting raw bioelectrical data in conjunction with BIA-derived body composition values when examining the effects of training and nutritional interventions.

\section{Acknowledgments}

The research was supported by a grant from Abbott Nutrition, Columbus, OH (ClinicalTrials.gov Identifier: NCT00798291).

\section{References}

Buffa, R., Saragat, B., Cabras, S., Rinaldi, A.C., Marini, E., 2013. Accuracy of specific BIVA for the assessment of body composition in the United States population. PLoS One 8, e58533.
Buffa, R., Mereu, E., Comandini, O., Ibanez, M.E., Marini, E., 2014. Bioelectrical impedance vector analysis (BIVA) for the assessment of two-compartment body composition. Eur. J. Clin. Nutr. 68, 1234-1240.

Dittmar, M., Reber, H., 2001. New equations for estimating body cell mass from bioimpedance parallel models in healthy older Germans. Am. J. Physiol. Endocrinol. Metab. 281, E1005-E1014.

Koury, J.C., Trugo, N.M., Torres, A.G., 2014. Phase angle and bioelectrical impedance vectors in adolescent and adult male athletes. Int. J. Sports Physiol. Perform. 9, 798-804.

Lukaski, H.C., 2013. Evolution of bioimpedance: a circuitous journey from estimation of physiological function to assessment of body composition and a return to clinical research. Eur. J. Clin. Nutr. 67 (Suppl. 1), S2-S9.

Lukaski, H.C., Hall, C.B., Siders, W.A., 2007. Assessment of change in hydration in women during pregnancy and postpartum with bioelectrical impedance vectors. Nutrition 23, 543-550.

Mackey, A.L., Esmarck, B., Kadi, F., Koskinen, S.O., Kongsgaard, M., Sylvestersen, A. Hansen, J.J., Larsen, G., Kjaer, M., 2007. Enhanced satellite cell proliferation with resistance training in elderly men and women. Scand. J. Med. Sci. Sports 17, 34-42.

Marini, E., Buffa, R., Saragat, B., Coin, A., Toffanello, E.D., Berton, L., Manzato, E., Sergi, G., 2012. The potential of classic and specific bioelectrical impedance vector analysis for the assessment of sarcopenia and sarcopenic obesity. Clin. Interv. Aging 7 585-591.

Marini, E.; Sergi, G.; Succa, V.; Saragat, B.; Sarti, S.; Coin, A.; Manzato, E.; Buffa, R. Efficacy of specific bioelectrical impedance vector analysis (BIVA) for assessing body composition in the elderly. J. Nutr. Health Aging 17:515-521; 2013

Micheli, M.L., Pagani, L., Marella, M., Gulisano, M., Piccoli, A., Angelini, F., Burtscher, M. Gatterer, H., 2014. Bioimpedance and impedance vector patterns as predictors of league level in male soccer players. Int. J. Sports Physiol. Perform. 9, 532-539.

Moon, J.R., Stout, J.R., Smith-Ryan, A.E., Kendall, K.L., Fukuda, D.H., Cramer, J.T., Moon, S.E., 2013. Tracking fat-free mass changes in elderly men and women using singlefrequency bioimpedance and dual-energy X-ray absorptiometry: a fourcompartment model comparison. Eur. J. Clin. Nutr. 67 (Suppl. 1), S40-S46.

Nescolarde, L.; Yanguas, J.; Medina, D.; Rodas, G.; Rosell-Ferrer, J. Assessment and followup of muscle injuries in athletes by bioimpedance: preliminary results. Conf. Proc. IEEE Eng Med Biol Soc 2011:1137-1140; 2011

Norman, K., Stobaus, N., Pirlich, M., Bosy-Westphal, A., 2012. Bioelectrical phase angle and impedance vector analysis-clinical relevance and applicability of impedance parameters. Clin. Nutr. 31, 854-861.

Norman, K., Wirth, R., Neubauer, M., Eckardt, R., Stobaus, N., 2015. The bioimpedance phase angle predicts low muscle strength, impaired quality of life, and increased mortality in old patients with cancer. J. Am. Med. Dir. Assoc. 16 (173), e117-e122.

Peterson, M.D., Sen, A., Gordon, P.M., 2011. Influence of resistance exercise on lean body mass in aging adults: a meta-analysis. Med. Sci. Sports Exerc. 43, 249-258.

Piccoli, A., Pastori, G., 2002. BIVA Software. Department of Medical and Surgical Sciences. University of Padova, Padova, Italy (available at e-mail apiccoli@unipad.it).

Piccoli, A., Rossi, B., Pillon, L., Bucciante, G., 1994. A new method for monitoring body fluid variation by bioimpedance analysis: the RXc graph. Kidney Int. 46, 534-539.

Piccoli, A., Codognotto, M., Piasentin, P., Naso, A., 2014. Combined evaluation of nutrition and hydration in dialysis patients with bioelectrical impedance vector analysis (BIVA). Clin. Nutr. 33, 673-677.

Saragat, B., Buffa, R., Mereu, E., De Rui, M., Coin, A., Sergi, G., Marini, E., 2014. Specific bioelectrical impedance vector reference values for assessing body composition in the Italian elderly. Exp. Gerontol. 50, 52-56.

Stout, J.R., Smith-Ryan, A.E., Fukuda, D.H., Kendall, K.L., Moon, J.R., Hoffman, J.R., Wilson, J.M., Oliver, J.S., Mustad, V.A., 2013. Effect of calcium beta-hydroxy-betamethylbutyrate (CaHMB) with and without resistance training in men and women 65 + yrs: a randomized, double-blind pilot trial. Exp. Gerontol. 48, 1303-1310. 